\title{
Constructing and Navigating Cultural Borderlands Using Augmentative and Alternative Communication
}

\author{
Martine M. Smith
}

Individuals who use augmentative and alternative communication (AAC) occupy complex sociocultural and sociolinguistic territories. They are immersed in spoken language communities but to participate as speakers in conversational interactions, they may rely on atypical communication modes, including systems for generating speech that set them apart as what C. Mattingly (2006) termed "Other." This article explores the challenges and tensions implicit in shifting between cultural roles of Natural Speaker and Aided Communicator using the construct of cultural borderlands. Borderlands are first explored in terms of social participation, focusing on questions such as whether aided communication offers a bridge across borderlands or constitutes a marker of cultural difference and on the question of ownership of aided communication. The borderlands of speaker-listener roles that are negotiated within interactions involving aided communication

[AQ1] are then considered. Possible implications of how the construct of cultural borderlands supports understanding of interactions involving aided communication are suggested. Key words: aided communication, borderland, communication device, speaker-listener roles, voice

$\mathbf{O}$ VER the past several decades, the field of augmentative and alternative communication (AAC) has expanded and evolved (e.g., Hourcade, Pilotte, West, \& Parette, 2004). Technological developments have opened up exciting possibilities to enhance communication access for individuals with severe communication impairments related to a broad range of disabilities. Augmentative and alternative communication solutions may serve many different purposes-to augment or replace speech for expressive communication, to support both comprehension and expres-

[AQ2]

[AQ3]
Author Affiliation: Department of Clinical Speech \& Language Studies, Trinity College Dublin, Dublin, Ireland.

The author declares no financial disclosure.

Corresponding Author: Martine M. Smith, PhD, Department of Clinical Speech \& Language Studies, Trinity College Dublin, 7-9 South Leinster St, Dublin 2, Ireland (mmsmith@tcd.ie).

DOI: $10.1097 / T L D .0000000000000148$ sion, and as a temporary or permanent set of resources and strategies.

The focus in this article is on people whom von Tetzchner and Martinsen (2000) characterize as using AAC for expressive purposes. Individuals in this group are presumed to be able to hear and understand spoken language but cannot produce expressive output that is sufficiently intelligible to meet their functional communication needs. Typically, individuals in this group require AAC over an extended period of time, if not permanently. Specifically, the focus here is on individuals who use aided communication, in other words communication that draws on resources external to the communicator. Such aided options can range from low-tech paper-based communication books and boards to high-tech speech-generating devices and computer-based systems. Although many in this group may experience some receptive language difficulties, their access to comprehension is primarily through spoken language. Their expressive communication 
is multimodal (as it is for most people), but uniquely, their multimodality incorporates use of aided communication.

Individuals who use aided communication for expressive purposes occupy complex sociocultural and sociolinguistic territories. They usually live their lives immersed in spoken language environments, in homes, families, and social networks, where their membership of cultural communities of "natural speakers" is unchallenged. However, for effective expressive communication, aided communication modalities may be required. The motor, cognitive, linguistic, and sensory demands of using aided communication create new interaction demands (e.g., Clarke \& Kirton, 2003; Light \& McNaughton, 2014; Murray \& Goldbart, 2009). The implicit rules of engagement that underpin successful spoken interactions (e.g., in terms of timing, linguistic specificity, or turn-taking) may be fractured, creating a new cultural territory that ranges from "not quite natural speaker" to "aided communicator."

Indeed, the complexity of the situation is partially reflected in the constant terminological debates and controversies about acceptable terms, labels, and descriptions (e.g., von Tetzchner \& Basil, 2011). Even the value of the term aided communicator is open to question, if the evidence is that for most individuals to whom this label is applied, communication relies more heavily on unaided than aided modalities (e.g., Andzik, Chung, \& Kranak, 2016). Such considerations have prompted questions about the appropriateness of talking about a sociolinguistic or sociocultural group defined by use of aided communication (e.g., Woll \& Barnett 1998). As noted by von Tetzchner (2016), "the presence of a communication aid may facilitate an attribution of identity as belonging to a category of aid users, but there may be a weak uniting force" (p. xi). However, if culture is defined as a form of encounter, rather than a form of life (Mattingly, 2008, p. 139), then the encounters of individuals who use aided communication with communication partners who use natural speech share sufficient overlap to suggest that culture may indeed of- fer a useful lens through which to view these experiences.

In Mattingly's terms, the practice of culture is [in part] "the practice of Othering, of identifying cultural (and racial) difference in a thousand subtle and unconscious ways" (2006, p. 495). Othering implies a focus on difference. In this respect, it is egocentric: The interpretation of "Other" is referenced back to the individual making a judgment, exploring "how is this person (the Other) different to $m e$ ?" Individuals who use aided communication may experience Othering in multiple ways across their days. This article will focus on practices of Othering at two levels: (a) the broader social context of everyday life and (b) the level of the individual, in the moment-bymoment context of interpersonal communication interactions. The construct of clinicalcultural borderlands is used to explore how individuals who use aided communication navigate their membership of cultural groupings, using as a starting point Mattingly's statement that "culture is not to be found in a group's shared set of beliefs and values so much as in its practices of drawing contrasts and boundaries with other groups" (Mattingly, 2006, p. 495). Cultural borderlands offer a uniquely rich context for considering culture, "in a borderland, culture emerges more vividly as a space of encounter than of enclosure" (Mattingly, p. 495). The metaphor of cultural borderlands offers a way of considering the interaction experiences of individuals who hear and understand spoken language but whose access to natural speech is severely restricted and who therefore find themselves between the worlds of natural speakers and aided communicators.

With the global rise of migration and displacement and the increasingly hostile rhetoric surrounding borders and geopolitical boundaries (http://www.unhcr.org/en-ie/ statistics/unhcrstats/5943e8a34/global-trends -forced-displacement-2016.html, retrieved December 15, 2017), the term borderland has a powerful resonance and significance. In Mattingly's terms, however, "border zones are not just places in which conflict and cultural 'Othering' take place. They are 
also places in which actors sometimes find common ground" (Mattingly, 2008, p. 139). They are spaces of encounter where people attempt to make themselves interpretable and to interpret the behaviors of others. For individuals who use aided communication, the struggle to be interpretable can play out overtly and covertly across the day, as they attempt to bridge the cultural divide between natural speaker and aided communicator and to make themselves readable in a way that is acceptable not only to their interaction partners but also to themselves.

[AQ4]

\section{CULTURAL BORDERLANDS AND AIDED COMMUNICATION}

Communication is one of the key means by which social groups connect, cultures coalesce, and shared understandings are negotiated. People with very limited expressive communication face significant barriers in asserting their membership of the spoken language cultural communities in which they are based. Because features of speech production are such salient markers of membership of a specific cultural group, failure to produce speech that marks that membership can have far-reaching consequences. Such Othering may be particularly salient for people whose understanding of the spoken language of their community is unimpaired, as well as people who are in the community physically, cognitively, and linguistically but marked out by their lack of speech as not of the community. One extreme example can be found in the autobiography of Martin Pistorius, who at the age of 12 developed locked-in syndrome due to an unexplained illness. Over the subsequent 5 years, he gradually regained cognitive awareness and receptive language skills. With no way of expressing himself that was interpretable to those he encountered, Pistorius struggled to bridge the cultural divide between the speaking communities in which he spent his days and the role assigned to him by virtue of his lack of speech. He described this role as a "box" marked in his words as "imbecile," a potted plant, "something to be given water and left in the corner" (Pistorius \& Davies, 2011, p. 16).

Aided communication offers a pathway into communication interactions. Such interactions serve as a foundation for social connections and belonging, for defining one's role within a community within a social network and ultimately, within a culture. However, this pathway may be fraught with challenges. Three such challenges are considered here. One challenge reflects the potential of aided communication to act as a barrier as well as a bridge to membership of the culture of natural speakers. The second challenge focuses on the importance of physical features of aided communication devices and the impact these may have on how easily someone may blend into a community of communication partners. The third challenge relates to fuzzy boundaries surrounding the role of aided communicator, the extent to which use of aided communication is required or expected, and the role of individuals in defining such boundaries.

\section{Communication aids: Markers of or bridges across cultural borders?}

In the writings of individuals who use aided communication, there are many powerful descriptions of the impact of having access to speech output in asserting identity and cultural membership. For example, Janice Staehely (2000) described an experience in a summer camp when she was 13 years of age. On her second day, a new counselor became frustrated after multiple efforts to put shoes on Janice's feet. Janice recalled:

[she] must have been thinking that I was a big, spoiled brat! I, on the other hand believed that the counselor was hopeless if she couldn't tell that the sneakers couldn't possibly be mine! ... . Finally I pointed to my [SGD] ...."Those are not my shoes" came out in a DEEP electronic male voice. There was a moment of silence and then laughter broke loose. Laughter was the music that started our friendship (Staehely, 2000, p. 8).

Despite the lack of concordance between the voice of the speech-generating device and Janice as a speaker, the act of speaking 
provided a bridge across the cultural boundary between Natural Speaker and Other and made it possible to negotiate common ground. This incident highlights the power that access to aided communication potentially offers in bridging cultural borderlands and finding ways to be interpretable. However, such success is not always easily achieved. The operational demands associated with aided communication can constitute significant barriers to successful and satisfying interactions.

Many individuals who use aided communication require additional support with many activities of daily living. As a group, they may experience more interactions with people paid to be in their presence (e.g., therapists, teachers, personal assistants) than with other kinds of communication partners (Thirumanickam, Raghavendra, \& Olsson, 2011). In their encounters with these partners, they may find themselves in a borderland between alternate identities as "Receiver of Help" and full conversational partner. In the study by Smith and Connolly (2008), many adults who used aided communication reflected on the time pressures they faced, including the difficulty of persuading care staff to pause long enough to allow genuine communication to take place. Being reliant on paid staff for activities such as washing, dressing, eating, and moving into the community, places individuals who use aided communication in a vulnerable power relationship within these encounters. They may juggle concerns about being perceived as a burden with a desire to establish a working relationship based on mutual understanding, an understanding that is normally negotiated through conversational interactions. Marion [pseudonym], who lives independently with the support of a number of personal assistants reflected in a personal communication (included here, with permission, in her own words, as typed by her):

talking is not comfortably if people only have time caring only for your physically needs. people might be in a hurry in doing other tasks in their job that they get too frustrated having a friendly conversation with you. ... You might be thinking of trying to make life easier for people assisting you as well as you. You dont mean to be a barrier to them. You fully understand there are many more more people to care about in their working environment.

For personal care assistants with busy schedules, the primary purpose of an encounter with an individual who uses aided communication may be the provision of care. Encounters that under other circumstances might offer a natural context for a conversation are transformed into new borderlands, where the pace and effort of interactions involving aided communication may be difficult to accommodate. Staff may compensate for these time pressures by avoiding conversations, occupying the conversational floor by maintaining monologues, or closing off the conversational space by maintaining silence (e.g., Smith, Murray, von Tetzchner, \& Langan, 2010). Individuals who persist in seeking interactions that are more satisfying are at risk of finding themselves labeled "difficult" or "demanding." The dilemma described by Marion was that she was aware of these pressures, but she also knew that a $\mathrm{g}$ ne conversation might lead to solutions that make life easier for everyone. That is, she knew that she could help her helper, rather than embodying the role of "barrier."

As illustrated by the experiences of Staehely (2000) and Pistorius \& Davies (2011) previously, access to aided communication can be a powerful tool for demonstrating eligibility for membership of the cultural community of natural speakers. However, as Marion's statement highlights, realizing this potential requires availability and capacity for engagement on the part of all people involved. There are also further potential costs to consider. The physical presence of a communication aid itself may create a new vulnerability to Othering, marking the speaker as visibly and acoustically different. The selection, design, and overall aesthetics of aided systems can therefore assume particular significance. 


\section{Aesthetics, appearances, and fitting in}

Many stakeholders may be involved in the process of selecting and designing an aided system for a specific individual. Often within those stakeholder groups, there are competing agendas, considerations, and pressures. These tensions can emerge in encounters that span multiple borderlands, between parents, education systems, clinical services, peer networks, parent-child interactions, and social community networks. Although all stakeholders may share common ground in viewing aided communication as a way of supporting effective participation for a child, adolescent, or adult, they may differ greatly in the values they place on features of an aided communication device, what a device should represent, or the purposes and roles they attribute to use of aided communication.

Stakeholders also may diverge in their expectations about whose voice carries most weight in decisions about device selection. Traditionally, recommendations about specific aided communication devices were often driven largely by the expertise of specialist speech-language pathologists, AAC specialists, or manufacturers, but the emergence of tablet technologies has revolutionized access to high-tech aided communication options (Fager, Bardach, Russell, \& Higginbotham, 2012; McNaughton \& Light, 2013). Not only are these options significantly more affordable, they also are more socially desirable. Augmentative and alternative communication specialists now find themselves in a new consumer-driven model of AAC technology provision (Hershberger, 2011), where families or individuals may arrive at an initial meeting with a preferred technological solution already in hand. Such encounters represent borderlands in which previously defined cultural roles such as Expert and Recipient/Pupil are fractured and new cultural roles must be negotiated.

In such interactions, the priorities of a speech-language pathologist may be focused not only on current needs but also on longterm goals related to attainment of communicative competence. From that perspective, key considerations may include how well a particular device or software application is likely to support language development or device longevity and availability of ongoing support. By contrast, parental concerns in selecting technology may be driven by priorities related to more immediate pressures of demonstrating competence, fitting in, and/or finding a niche. Whereas using a speech-generating device may mark a child as Other, using an iPad may allow for a different status of Other, even a desirable Other. In her book, Giving Voice, Alper (2017) tracked the experiences of 20 families parenting young people who used iPads to support their communication. Many of the parents mentioned that while their children were socially ostracized in multiple ways, they were more easily accepted among peers because of the appeal of their iPad. Danny's mother Alice observed "A person sees someone like Danny with an iPad and it's not weird" (Alper, 2017, p. 73).

In Alper's (2017) research, families were concerned about how the physical appearance of a device reflected the presentation of self for their family members. They were keen to reduce the risk of an aided communication device creating potential borders, marking their family member out as Other. These concerns extended to the case purchased for an iPad. Many families spent considerable time researching optimal cases that offered protection but also flexibility, accessibility, and appeal to the wider community as a way of blending in. However, the parents interviewed by Alper perceived that schools purchasing iPads for students often attended only to the need to safeguard the iPad, regardless of what the visual features of a protective case might say about an individual child. For example, a parent called David expressed his frustration about a new black iPad case purchased by the school, and what he felt the case communicated about his daughter, Beatriz. To him, the new case sent out a loud and clear message about his daughter "She's careless, beware now, she's going to drop it, she's going to break it" (Alper, 2017, p. 76). From this perspective, the emphasis on protecting the 
iPad sabotaged its potential to reduce barriers and reinforced a narrative that depicted personal incompetence, flying in the face of the stated purpose of the iPad to enhance communicative competence.

Some of these tensions may be understood in terms of the financial implications related to aided communication. Provision of a "voice" for individuals who use aided communication costs money. These costs can amount to several thousands of dollars, putting individuals who use aided communication in a uniquely vulnerable situation as they try to navigate a path to participation. They and their families may find themselves cast in the role of "Beneficiary," disempowered and reluctant to challenge decisions or recommendations for fear of losing what may be a long-awaited opportunity. For example, Daisy, a mother in Alper's (2017) study, was unhappy with some of the decisions made by her son's school about the purpose of the iPad but recognized the imbalance in power in their relationship. She reasoned, "I shouldn't complain too much. Just the fact that they gave me an iPad, and the fact that I'm able to put stuff for home and that it's just his and that we get to take it home" (2017, p. 99). The cost implications raise complex questions about ownership of communication devices and obligations related to the role of being a Beneficiary.

\section{Obligations, ownership, and autonomy:} Whose voice is it anyway?

Not all costs associated with aided communication are clearly visible. The apparent low costs of communication boards mask the time costs involved in determining the appropriate symbolic representational system, the target vocabulary, and the optimal display organization. Indeed, these costs (both the upfront purchase cost of a high-tech device and hidden costs of low-tech options) are simply the first installment in an investment process that may extend over many months or years in attempts to optimize a system for a specific individual.

Aided communication may assume a status and importance as a key mode of communi- cation, despite the evidence that (a) effective communication is multimodal (Light $\&$ McNaughton, 2014), (b) unaided modes such as facial expression, eye gaze, gesture, and vocalization are frequently more efficient, more accessible, and more transparent than aided communication (Loncke, 2014), and (c) unaided modes are frequently relied on even by individuals who are considered expert aided communicators (e.g., Joyce, 2000; Williams, 2000). As a Beneficiary of a high-cost device that has taken time, effort, and money to procure, an individual may either assume or be assigned a range of obligations, including an obligation to use what has been provided as effectively as possible, as much as possible, for as many purposes as possible, in order to provide evidence that the investment was worthwhile.

One immediate problem is that there is no clear standard against which "worthwhileness" can be measured. How much use of aided communication is enough? Is frequency of use the only valid measure? Terms such as device "abandonment" or "underutilization" (Johnson, Inglebret, Jones, \& Ray, 2006) carry connotations of waste or of failure, even though, as pointed out by Scherer (2005), a device may be categorized as abandoned because someone has developed skills to move on successfully to a more complex piece of technology. Some of the adults interviewed by Smith and Connolly (2008) rated their use of aided communication as infrequent but nonetheless described their aided communication as essential, often because the instances when they needed their aided communication involved high-stakes interactions.

An aided communication system that fits well with the communication needs and personal requirements of an individual and his or her key communication partners is likely to be used more frequently and to become truly functional. However, finding such optimal alignment can take time. Even when it is achieved, aided communication may be only one option for expressive communication. Unaided modes of communication may be less fatiguing and at times, more efficient 
and interpretable. Unaided modes also may allow individuals to assert alignment with others, to identify themselves as members of the interaction community, and to belong to the cultural group of natural speakers.

It is difficult to imagine a situation in which individuals who use natural speech could be required to justify the extent to which they use gesture, facial expression, or intonation. A tension in relation to aided communication is that it takes time and practice to master the operational demands of a communication aid. Individuals need access to aided communication systems that are relevant to their needs and abilities, as well as multiple opportunities to use aided communication in order to achieve the level of competence that allows them to make informed choices about their preferred modes of communication. Negotiating that process requires crossing a borderland where boundaries around ownership of communication modes and autonomy in communication preferences may easily be blurred. There may be sound reasons why an individual is explicitly requested to use aided communication, even if a message has already been interpreted and understood (e.g., Smith, 2003).

[AQ6] However, if aided communication ultimately is to enhance personal autonomy and choice, then ownership of modes of communication also implies choices about when not to use a communication device. Staehely (2000, p. 5) recalled

despite all the hard work at practicing to use my language board, I think I always left it at home. In my opinion, it was much easier to use my own voice or to rely on my mom, sisters, or close friends to say my words for me.

Similarly, Estrella (2000) challenged the perceived obligation to use his device in a prespecified way. Messages can be stored in a device as single words or, alternatively, as longer stretches of speech such as phrases, sentences, or extended paragraphs of output. Prestored utterances can be generated with a single-cell selection and thereby increase rate of communication and potentially enhance overall communicative efficiency. However, as Estrella pointed out, prestored utterances are often a poor fit with immediate communication demands:

I was also given piles of sentences and criticized for not using them. I don't know about you, but I don't think in terms of pre-formed sentences. Sometimes I even change my thought halfway through the sentence and $I$ have also been known to throw in a very descriptive word when the mood strikes me. I can think of few things more dehumanizing and even demeaning than selecting canned sentences from a list and seeing the subject matter that you want to talk about is nowhere to be found (p. 38).

Ownership can be further blurred by complications about the funding source. On the one hand, individuals may be directed to "Use your device," but on the other hand, decisions about access to the device may ultimately be held by others. If a device is purchased by a school district for a specific student, then the district may control not only the case or other ancillary products provided but also access to the device as in the case of Daisy's son described earlier. In contexts of low income, devices may be shared across multiple students and their use limited to the school context (Tönsing \& Dada, 2016). In other contexts, the demarcations may be less extreme but no less present because of their subtlety. For example, in some instances, damage to a device may be covered only by insurance if the damage is incurred on school property, implying that the rightful place for a device is geographically defined rather than linked to an individual (Alper, 2017). Some of the parents in Alper's (2017) study objected to the decision of school districts to lock an iPad into a communication application without consultation with family members or students. In the case of one student, Beatriz, a new passcode on the iPad effectively locked her out of what was on the one hand her communication device, with all its associated obligations, but simultaneously the property of the school. As her father commented:

Beatriz was free to transport the mobile device back and forth between home and school, but both 
the device and her communicative agency were immobilized by the school's device settings and technology management infrastructure once she walked through the doors of her home. Along the way, both humans and machines made decisions that rendered Beatriz's iPad simultaneously, and irreconcilably, mobile and immobile (Alper, 2017, p. 101).

The complications related to ownership and obligation, outlined previously, speak to the fluid and blurred boundaries between natural speaker and aided communicator and the complicated territory between aided communicator and Not-Quite Natural Speaker roles. These tensions also play out at the level of individual, interpersonal conversational interactions.

\section{CULTURAL BORDERLANDS WITHIN MULTIMODAL INTERACTIONS}

Interactions that involve use of aided communication are often described as differing structurally and temporally from other interactions (e.g., Clarke \& Kirton, 2003; Light, 1988; von Tetzchner \& Stadskleiv, 2016). The pace and rhythm of interactions are influenced by the demands of accessing and selecting messages within an aided communication system (Clarke \& Wilkinson, 2008; Higginbotham, Fulcher, \& Seale, 2016). Communication partner roles may be extended to disambiguate contributions communicated using aided communication (e.g., Bloch \& Clarke, 2013). Within these conversations, individuals who use aided communication occupy roles as both speaker and listener. In their roles as listener, their membership of the natural speaker community is unchallenged. However, in their role as speaker, they are vulnerable to Othering. This vulnerability may be increased by the pace and dynamics of interactions where aided contributions must first be individually selected and sequenced. Individuals then often need to explicitly negotiate shared interpretations of aided output so that the conversational task of "narrative mind reading" (Mattingly, 2008, p. 136) may be particularly complicated for all involved.

\section{Conversation pacing and structure}

Within these conversational encounters, the complexity of multimodal communication plays out in real time, influencing the role of aided communicator as speaker but also challenging the traditional role of listener for the person's communication partner. If in cross-cultural encounters, "individuals are continually engaged in creating plausible narratives to make sense of the exotic actions of others" (Mattingly, 2008, p. 139), then from the point of view of the listener, the exotic action of a speaker who makes no apparent response is plausibly interpreted within a narrative of rudeness, of incompetence, of disengagement, or of boredom. Such misunderstandings can easily occur while someone attempts to use a scan-dependent, speechgenerating device or pauses to consider how to communicate something when the necessary vocabulary is not available. Pistorius and Davies (2011) reflected eloquently on the impact of aided communication on the structure of his conversations and, by implication, his role as a speaker:

A conversation with me is slow and takes time and a patience that many people don't have ... people's voices move in a seamless cycle, sentences running one into another while they talk. But I interrupt the rhythm and make it messy. People must make a conscious effort to look at me and listen to what I have to say. They must allow me the space to speak because I can't butt in and many don't want to listen to the silence I create as they wait for me to input words into a computer ... a conversation with me is as much about the silences as it is about the words, and I notice if my words are listened to or not because I choose each one so carefully. (p. 139)

Even in simulated conversations between individuals without disabilities, where use of aided communication is contrived, individuals have been found to adapt their communication style to accommodate the unique demands of generating and interpreting aided contributions (Farrier, Yorkston, Marriner, \& Beukelman, 1985; Smith, McCague, O'Gara, \& Sammon, 2016). For example, the college students in the study by Smith et al. (2016) 
reflected on the responsibilities they felt as natural-speaking partners for managing the overall flow and success of the interactions. Comments included "I talked more to keep the conversation going and to make it easier for the person (P1); I felt like I should fill in the blanks for my partner when they couldn't find the word (P2)" (p. 273). When they switched roles to use aided communication, they reported a sense of pressure, of making each symbol selection count to minimize the burden on their naturally speaking partner (e.g., "I was able to convey most factual information but I felt like I couldn't tell a story or write long sentences" (P1); "I started limiting myself to bare essentials of answering questions without expanding" (P4); "I felt I had to stick to topics I could see on the board" (P4); p. 280).

Added to these challenges are the cognitive and physical efforts often involved in both generating and interpreting aided communication, where misunderstandings are common, and fatigue and frustration can quickly transform a conversation into a chore. One stark example is described by Higginbotham et al. (2016), where Rita, a 49-year-old woman, who used a speech-generating device using a head mouse and knee switch, was interacting with her husband, Max, to complete a way-finding task. Over the course of the task, Rita experienced several technical problems while composing her utterances, resulting in long delays in generating a response. Max, apparently unaware of these issues and unable to interpret the apparently "exotic action" of no response, expressed his frustration by directing her to not "... spell out the whole thing, just one WORD in there. One letter man" (p. 215). As pointed out by the authors, Max's words imply that Rita, rather than the device, was responsible for the communication delays. In this interaction, Rita was "Othered," in the sense that Max seemed to judge her either as not aware of the temporal demands of effective conversations or unwilling to comply. Citing the work of Robillard (1994), Higginbotham et al. (2016) reflected that "finding fault with the augmented speaker in not adhering to the temporal order of conversation is common practice, and, unfortunately, one which frequently brings with it notions of incompetence and reluctance to maintain long-term relationships" (p. 215).

\section{The question of voice in voice output}

Although speech-generating devices may be promoted as the "voice" of individuals with severe speech impairments, the status of voice output within interactions may be somewhat uncertain. An important question is whether such output should be literally interpreted as the "voice" of someone who uses aided communication, or as a representation of voice more generically. For example, Cormac, aged 8 years, used a speech-generating device to describe to his mother what had happened in a video he had viewed (see Table 1).

Cormac's mother could not see the visual display of his communication device and at Line 4 misinterpreted "laughed" as "left." At Line 8, Cormac attempted to repair this breakdown, using gesture. His gestures were interpreted as ambiguous, potentially mocking his mother, and she directed him to "tell me," an explicit ding to use speech. He complies, but whe makes the device visible to his mother, she referred to "the voice" (Line 17) rather than Cormac's voice, implying that it belongs to a third party-in her words "that bloody [device]." This designation granted permission to overtly evaluate the voice negatively in a way that would be unacceptable if it were conceived as that of a natural speaker. This interaction illustrates the potential paradox of being in the role of a speaker, generating a voice that is somehow not quite the voice of the speaker.

One possible strategy to support navigation of this complex terrain of conversational shifts in roles of speakers and listeners is to focus on the shared task of conducting conversations, instead of designating one participant as the aided communicator. Clarke and Wilkinson (2008) proposed that rather than Othering the individual who has the 
Table 1. Extract of conversation describing contents of silent video event

\begin{tabular}{|rll|}
\hline & Speaker & \\
\hline 1 & Cormac & "Three" "three people" \\
2 & Mother & Yeab? \\
3 & Cormac & "Three people were jumping in to a pool the two laughed" \\
4 & Mother & What do you mean, two left? \\
5 & & Three people jumped in and two got out? \\
6 & Cormac & Hub? \\
7 & Mother & Only one jumped in? And the other two took off? \\
8 & Cormac & "Laughing" (points and extends index finger, copying action of \\
& Mother & Go on, tell me! I can't guess with that. \\
9 & Cormac & That just looks like as if you're laughing at me \\
10 & Mother & Yeab, I beard that bit. \\
12 & & What do you mean the (mimics action of laughing). \\
13 & & What does that mean? \\
14 & Cormac & "They laughed" (turns device to show mother) \\
15 & Mother & They laughed, sorry. \\
16 & & That's that bloody [names device], the voice is desperate. \\
17 & & They laughed so they were jumping in the pool \\
18 & &
\end{tabular}

responsibility for aided communication, discussions should center on interactions that involve aided communication. This would shift the emphasis to all participants in negotiating meaning as part of a common endeavor. Through this lens, interpretation of aided communication becomes a shared space for participants. Despite the new understandings this perspective offers, questions of voice, ownership, and authorship resonate particularly in the field of aided communication, in light of controversies such as Facilitated Communication or the Rapid Prompting Method (see Schlosser et al., 2014; Tostanoski, Lang, Raulston, Carnett, \& Davis, 2014; Travers, Tincani, \& Lang, 2014). At the core of these controversies are questions related to autonomy in communication and blurring of boundaries between speakers' and listeners' roles and responsibilities. It seems likely, therefore, that voice output will continue to occupy a curious disembodiment within interactions, belonging to-but not quite owned by-one of the participants within the interaction.
According to Mattingly (2008), "culture has come to be the name of a land one travels through as much as lives in, a land characterized by hybridity" (p. 139). In the many and varied contexts in which people who use aided communication navigate their daily lives and conversations, they encounter strangers and familiars. In the complex cultural borderlands between "natural speaker," "not quite natural speaker," and "aided communicator," both their achievements and their challenges can be exposed. The decisions of other players in these cultural borderlands can exert a powerful influence on their conversational experiences and on their success in bridging the apparent divides between these multiple identities. The construct of cultural borderland offers a valuable prism through which to understand some of the challenges they encounter in constructing identities as communicators.

To some extent, recognizing these challenges as cultural as well as operational or linguistic offers new ways of influencing change. At a basic level, recognizing the 
vulnerability and risk of social exclusion of individuals with significant communication challenges highlights the importance and potential power of aided communication in asserting competence and membership of an interaction community. Explicit discussion of the ways in which aided communication may not only support membership of that community but also create barriers offers a mechanism to increase awareness, tolerance, and openness to communication diversity that can support richer communication interactions for all concerned. Following Clarke and Wilkinson's (2008) suggestion that the focus should shift away from individuals who use aided communication to interactions that involve aided communication creates a new way of considering what interventions might target. Specifically, interventions may target development of an individual's skills and competencies or extending capacities within dyads to engage in effective "narrative mind reading" (Mattingly, 2008, p. 136).

Cultural borderlands are relatively unchartered and constantly changing. They evolve and emerge in cultural practices. As such, they offer opportunities for new understandings across a range of domains. Aided communication is a relatively recent phenomenon and its reach is broad and diverse. Communication aids can be regarded as cultural artifacts, whose value is defined through use within a cultural context. In this sense, the construct of cultural borderlands offers a potentially valuable framework for exploring both the cultural practices and values associated with aided communication.

\section{REFERENCES}

Alper, M. (2017). Giving voice: Mobile communication, disability and inequality. Cambridge, MA: The MIT Press.

Andzik, N., Chung, Y., \& Kranak, M. (2016). Communication opportunities for elementary school students who use augmentative and alternative communication. Augmentative and Alternative Communication, 32, 272-281. doi:10.1080/07434618.2016. 1241299

Bloch, S., \& Clarke, M. (2013). Handwriting-in-interaction between people with ALS/MND and their conversation partners. Augmentative and Alternative Communication, 29, 54-67. doi:10.3109/07434618. 2013.767497

Clarke, M., \& Kirton, A. (2003). Patterns of interaction between children with physical disabilities using augmentative and alternative communication and their peers. Child Language Teaching and Therapy, 19, 135-151.

Clarke, M., \& Wilkinson, R. (2008). Interaction between children with cerebral palsy and their peers 2: Understanding initiated VOCA-mediated turns. Augmentative and Alternative Communication, 24, 3-15.

Estrella, G. (2000) Confessions of a blabber finger. In M. Fried-Oken \& H. Bersani (Eds.), Speaking up and spelling it out (pp. 31-46). Baltimore, MD: Paul H. Brookes.

Fager, S., Bardach, L., Russell, S., \& Higginbotham, D. J. (2012). Access to augmentative and alternative communication: New technologies and clinical decision-making. Journal of Paediatric Rehabil- itation Medicine, 5, 53-61. doi:10.3233/PRM-2012 $-0196$

Farrier, L., Yorkston, K. M., Marriner, N., \& Beukelman, D. (1985). Conversational control in nonimpaired speakers using an augmentative communication system. Augmentative and Alternative Communication, 1 , 65-73.

Hershberger, D. (2011). Mobile technology and AAC apps from an AAC developer's perspective. Perspectives on Augmentative and Alternative Communication, 20, 28-33. doi:10.1044/aac20.1.28

Higginbotham, D. J., Fulcher, K., \& Seale, J. (2016). Time and timing in interactions involving individuals with ALS, their unimpaired partners and their speech generating devices. In M. Smith \& J. Murray (Eds.), The silent partner? Language, interaction and aided communication (pp. 199-229). London: J\&R Press.

Hourcade, J., Pilotte, T., West, E., \& Parette, P. (2004). A history of augmentative and alternative communication for individuals with severe and profound disabilities. Focus on Autism and Other Developmental Disabilities, 19, 235-244. doi:10.1177/10883576 040190040501

Johnson, J. M., Inglebret, E., Jones, C., \& Ray, J. (2006). Perspectives of speech language pathologists regarding success versus abandonment of AAC. Augmentative and Alternative Communication, 22, 85-99.

Joyce, M. (2000). A fish story. In M. Fried-Oken \& H. Bersani (Eds.), Speaking up and spelling it out (pp. 87-96). Baltimore, MD: Paul H. Brookes. 
Light, J. (1988). Interaction involving individuals using augmentative and alternative communication: State of the art and future directions. Augmentative and Alternative Communication, 4, 66-82.

Light, J., \& McNaughton, D. (2014). Communicative competence for individuals who require augmentative and alternative communication: A new definition for a new era of communication? Augmentative and Alternative Communication, 30, 1-18.

Loncke, F. (2014). Augmentative and alternative communication: Models and applications for educators, speech-language pathologists, psychologists, caregivers and users. San Diego, CA: Plural Publishers.

Mattingly, C. (2006). Pocahontas goes to the clinic: Popular culture as lingua franca in a cultural borderland. American Anthropologist, 108, 494-501.

Mattingly, C. (2008). Reading minds and telling tales in a cultural borderland. Ethos, 36, 136-154. doi:10.1111/j.1548-1352.2008.00008.x

McNaughton, D., \& Light, J. (2013). The iPad and mobile technology revolution: Benefits and challenges for individuals who require augmentative and alternative communication. Augmentative and Alternative Communication, 29, 107-116. doi:10.3109/074 34618.2013.784930

Murray, J., \& Goldbart, J. (2009). Cognitive and language acquisition in typical and aided language learning: A review of recent evidence from an aided communication perspective. Child language Teaching and Therapy, 25, 31-58. doi:10.1177/02656590080 98660

Pistorius, M., \& Davies, M. L. (2011). Ghost boy. London: Simon \& Schuster.

Robillard, A. (1994). Communication problems in the intensive care unit. Qualitative Sociology, 3, 11971121.

Scherer, M. (2005). Living in the state of stuck: How assistive technology impacts the lives of people with disabilities (4th ed.). Cambridge, MA: Brookline Books.

Schlosser, R., Balandin, S., Hemsley, B., Iacono, T., Probst, P., \& von Tetzchner, S. (2014). Facilitated communication and authorship: A systematic review. Augmentative and Alternative Communication, 30, 359-368. doi:10.3109/07434618.2014.971490

Smith, M., \& Connolly, I. (2008). Roles of aided communication: Perspectives of adults who use AAC. Disability and Rebabilitation: Assistive Technology, 3, 260-273. doi:905849173 [pii]10.1080/174831 00802338499

Smith, M., McCague, E., O'Gara, J., \& Sammon, S. (2016). "...this is not going to be like, you know, standard communication?": Naturally speaking adults using aided communication. In M. Smith \& J. Murray (Eds.), The silent partner: Language and interaction in aided communication (pp. 269-288). London: J\&R Press.
Smith, M., Murray, J., von Tetzchner, S., \& Langan, P. (2010). A tale of transitions: The challenges of integrating speech synthesis in aided communication. In J. Mullenix \& S. Stern (Eds.), Computer synthesized speech technologies: Tools for aided impairment (pp. 234-256). New York, NY: IGI Global.

Staehely, J. (2000). The communication dance. In M. Fried-Oken \& H. Bersani (Eds.), Speaking up and spelling it out (pp. 1-12). Baltimore, MD: Paul H. Brookes.

Thirumanickam, A., Raghavendra, P., \& Olsson, C. (2011). Participation and social networks of schoolage children with complex communication needs: A descriptive study. Augmentative and Alternative Communication, 27, 195-204. doi:10.3109/ 07434618.2011.610818

Tönsing, K., \& Dada, S. (2016). Teachers' perceptions of implementation of aided AAC to support expressive communication in South African special schools: A pilot investigation. Augmentative and Alternative Communication, 32, 282-394. doi:10.1080/07434618.2016.1246609

Tostanoski, A., Lang, R., Raulston, T., Carnett, A., \& Davis, T. (2014). Voices from the past: Comparing the rapid prompting method and facilitated communication. Developmental Neurorebabilitation, 17, 219-223.

Travers, J., Tincani, M., \& Lang, R. (2014). Facilitated communication denies people with disabilities their voice. Research and practice for persons with severe disabilities, 39, 195-202. doi:10.1177/ 1540796914556778

von Tetzchner, S. (2016). Foreword. In M. Smith \& J. Murray (Eds.), The silent partner? Language, interaction and aided communication (pp. ix-xii). London: J\&R Press.

Von Tetzchner, S., \& Basil, C. (2011). Terminology and notation in written representations of conversations with augmentative and alternative communication. Augmentative and Alternative Communication, 27, 141-149. doi:10.3109/07434618.2011.610356

von Tetzchner, S., \& Martinsen, H. (2000). Introduction to augmentative and alternative communication (2nd ed.). London: Whurr.

von Tetzchner, S., \& Stadskleiv, K. (2016). Constructing a language in alternative forms. In M. Smith \& J. Murray (Eds.), The silent partner? Language, interaction and aided communication (pp. 17-34). London: J\&R Press Ltd.

Williams, M. B. (2000). Just an independent guy who leads a busy life. In M. Fried-Oken \& H. Bersani (Eds.), Speaking up and spelling it out (pp. 221-230). Baltimore, MD: Paul H. Brookes.

Woll, B., \& Barnett, S. (1998). Toward a sociolinguistic perspective on augmentative and alternative communication. Augmentative and Alternative Communication, 14, 200-211. doi:10.1080/0743461 9812331278376 


\section{Queries to Author}

Title: Constructing and Navigating Cultural Borderlands Using Augmentative and Alternative Communication

Author: Martine M. Smith

[AQ]: Please check if authors name are correctly captur (CD given names (in red) and surnames (in blue) for indexing after publicatio

[AQ1]: Please check whether the key words are $\mathrm{OK}$ as iypeset

[AQ2]: Please check whether the author affiliation is OK as typesen.

[AQ3]: Please check whether the corresponding address $\Omega_{K}$ as tyeant.

[AQ4]: Please check whether the levels of headings are OK.

[AQ5]: Please check whether the 1 (G) running head is OK as typeset.

[AQ6]: Please include references "ImIth, 2003" and "Smith et al. (2016)" in the list of references. 